\title{
Minimizing the Average Delay at Intersections via Presignals and Speed Control
}

\author{
Mina Ghanbarikarekani $\mathbb{D}^{1},{ }^{1}$ Xiaobo Qu $\left(\mathbb{D},{ }^{2}\right.$ Michelle Zeibots, ${ }^{3}$ and Weiwei Qi ${ }^{4}{ }^{4}$ \\ ${ }^{1}$ School of Civil and Environmental Engineering, University of Technology Sydney, Sydney, NSW 2007, Australia \\ ${ }^{2}$ Department of Architecture and Civil Engineering, Chalmers University of Technology, Gothenburg, Sweden \\ ${ }^{3}$ Institute for Sustainable Futures, University of Technology Sydney, Sydney, NSW 2007, Australia \\ ${ }^{4}$ School of Civil Engineering and Transportation, South China University of Technology, Guangzhou 510641, China
}

Correspondence should be addressed to Weiwei Qi; qwwhit@163.com

Received 27 January 2018; Accepted 8 April 2018; Published 29 May 2018

Academic Editor: David Z. W. Wang

Copyright ( 2018 Mina Ghanbarikarekani et al. This is an open access article distributed under the Creative Commons Attribution License, which permits unrestricted use, distribution, and reproduction in any medium, provided the original work is properly cited.

\begin{abstract}
Urban intersections have been well recognized as bottlenecks of urban transport systems. It is thus important to propose and implement strategies for increasing the efficiency of public and private transportation systems as a whole. In order to achieve this goal, an additional signal could be set up near the intersection to give priority to buses through stopping vehicles in advance of the main intersection as a presignal. It has been increasingly popular in urban cities. While presignals indeed reduce the average delay per traveler, they cause extra stops of private vehicles, which might compromise the overall efficiency, safety, and sustainability. This paper aims to propose a model to improve presignals by reducing the vehicles' number of stops behind the presignals. By applying the method, vehicles would be able to adjust their speed based on traffic conditions as well as buses' speed and approach. Numerical analyses have been conducted to determine the conditions required for implementing this method.
\end{abstract}

\section{Introduction}

Traffic congestion is one of the most significant issues in big cities that dissatisfy inhabitants. This matter has remarkably inevitable impacts on people's daily life in terms of travel time and health that resulted from increasing delay, traffic congestion, and air pollution [1-4]. Therefore, promising methodologies, which mitigate traffic congestion, play an important role in improving the life situation and transportation systems in main cities, specifically in their central business district [5]. Public transportation plays a vital role in decreasing traffic congestion as it has higher passenger factor than private vehicles, while it occupies less road space [6]. Hence, one of the most efficient ways that results in calming traffic congestion is developing public transit and encouraging people to use it rather than their private vehicles [7]. To serve this purpose, it is necessary to enhance the performance of public transportation systems in terms of delay and travel time.

Traffic control strategies often prioritize public transportation to motivate people not to use their private vehicles
[8-10]. Presignaling for buses has been proven one of the effective strategies to reduce the average delay per passenger [11]. Presignal is a kind of traffic signal that is implemented in advance of main signalized intersections and provides priority for buses in choosing their approach behind the main intersections by giving red signal to private vehicles. Although this method has remarkably efficient effects on reducing buses' delay and travel time and increasing their speed, it has several disadvantages in traffic flow. More specifically, presignals impose additional stops to private cars that lead to the increase of their delay and travel time. In other words, using presignals enhances the functionality of buses through prioritizing them at intersections, while it does not consider private vehicles.

This study aims to give priority to buses as well as improve the performance of the private vehicles through minimizing their number of stops while reaching presignals. For the sake of alleviating the cars' number of stops, it is suggested to amend their speed based on traffic signal timing, buses approach, and traffic condition ahead. Consequently, 
the traffic flow of the private cars will be continuous and balanced rather than being discrete.

The rest of the paper is structured as follows. Section 2 explains background studies carried out related to the topic. Section 3 describes the methodology, the essential assumptions, and its procedure. Numerical analysis of the model, the results, and discussion are presented in Section 4. Section 5 shows the conclusion.

\section{Literature Review}

Presignalizing is one of the most recent innovations that have been paid attention over the last two decades. The bus presignal was defined by Peake [12] as the traffic signals implemented behind intersections for managing traffic flow and giving buses priority. The first time using presignals for prioritizing buses was proposed by $\mathrm{Wu}$ and Hounsell [13]. In their research, three different categories were presented including (1) implementing presignals with buses uncontrolled, (2) presignals with controlling both buses and cars, and finally (3) giving the red signal to private vehicles during arriving buses to presignal and then giving the red signal to the bus lane. It needs to be mentioned that, according to studies conducted by $\mathrm{Wu}$ and Hounsell [13], Kumara and Hounsell [14], He et al. [15], Xuan [16], and Xuan et al. [17], using and implementing presignals upstream of the urban intersections lead to the decrease of the discharge rate of the intersections and the waste of the intersection's green time. In other words, fewer vehicles can be discharged from presignals than the capacity and green time of the main intersection.

Kumara and Hounsell [14] proposed queue relocation to avoid wasting the main intersections' green time. Two priority methods in presignalized intersections, queue relocation and bus priority, were suggested in their research. Queue relocation keeps private vehicles at presignal stop line due to saving the green time of the main intersection. Moreover, bus priority is supplied by detectors embedded in vehicles. They indicated that presignals play a remarkable role in prioritizing buses in oversaturated intersections throughout queue relocation and bus priority.

Another solution offered to solve the problem of intersections' discharge rate is using mid-block presignals posed by Xuan et al. [17]. They demonstrated that this solution causes traffic flow to be stored efficiently between presignals and main intersections. Moreover, for increasing the intersections' discharge rate, an adaptive algorithm was proposed by He et al. [15]. This algorithm can control presignals regarding real-time demand for private and public transportation. It was achieved by accurate statistics and real-time detection. They indicated that presignals with adaptive control algorithm lead to the stimulation of the buses' use and reduction of person delay more than continuous and interrupted bus lane strategies, respectively. Increasing the intersections flow capacity is one of the significant parameters that affects the intersections efficiency fundamentally. Another considerable study carried out for increasing the intersections' capacity is Xuan's research [16]. Xuan has proposed a method in order to raise the signalized intersections flow capacity using tandem design. In this study, left-turning and through-moving vehicles were sorted throughout a mid-block presignal. Xuan conducted studies on the requirements of the blocks' length for reaching the optimal capacity and the effects of the tandem design on minimizing these requirements. It was shown that this tandem design had increased the flow capacity of cars and buses in intersections equipped with presignals.

In addition to the low discharge rate of the intersections, $\mathrm{Wu}$ and Hounsell [13] have explained another issue of presignals in their research and that is an extension of queue length to the upstream intersection. They have suggested vital assumptions for estimating traffic signal timing to avoid these mentioned issues.

Kejun [18] has conducted a study about prioritizing buses at a single intersection using presignal and passive priority through introducing Bus Advance Area between stop lines of the presignalized and main intersections. In addition, Kejun investigated the efficiency of presignal by simulating the studied intersection in VISSIM; consequently, it was demonstrated that although presignals increase the efficiency of the buses, they have a negative impact on the private vehicles' performance because of their additional stop behind presignals. For eliminating this impact at intersections equipped with presignal, He et al. [19] have suggested a control algorithm with an online performance. This algorithm has been modelled in microsimulation software, VISSIM. In their research, they implemented the algorithm with the bus lane, mixed lane, and presignal strategies. In the end, by comparing these methods, it was demonstrated that using presignals with the proposed algorithm not only prioritizes buses but also maintains proficiency of the private vehicles. In addition to He et al's studies, Guler and Menendez have played a significant role in improving the functionality of private vehicles as well as bus priority. Guler and Menendez [20] have estimated delays of cars and buses in presignalized intersections analytically using queuing theory. In their research, they have computed traveler delays in implemented presignals and allocated a lane to buses. By comparing the commuters' delay, it was concluded that presignal systems minimize the delay more than dedicated bus lanes. Consequently, implementing presignals has fundamental effects on buses due to their high capacities. In addition, Guler and Menendez [21] have presented a practical instruction about using presignals upstream of the intersections. The influences of implementing presignal on intersections comparing to other bus priority strategies pave the way to determine the conditions of applying presignals in arterials. It should be mentioned that they have proposed the presignals instruction to improve transit services and private transportation systems simultaneously. Furthermore, Guler et al. [22] have suggested an innovative strategy to prioritize buses as well as improving the functionality of cars. This strategy provides dynamic timing, for presignals, which can be activated or deactivated depending on the traffic situation. In their research, it has been considered that implementing presignals leads to increasing cars' delay in undersaturated intersections and reducing their discharging rate in oversaturated intersections. However, using the single-lane strategy can mitigate the mentioned undesirability. 


\section{Methodology}

In this section, we first present some essential assumptions for the model. We further describe the model and its procedures in Section 3.2. In addition, the model's objective function based on the initial queue delay and the required parameters for the model are elaborated.

3.1. The Model Assumptions. In this study, there are some assumptions notified for proposing and presenting a model:

(i) Without loss of generality, we assume there are two lanes.

(ii) The intersection is controlled by a fixed-time traffic signal and equipped with presignal.

(iii) It is assumed that the studied intersection is isolated; that is, the performance of the adjacent intersections has no influence on it.

(iv) A Variable Message Sign (VMS) is installed for alarming the private cars.

(v) The Automatic Vehicle Location system (AVL) is utilized in each bus for the sake of determining the buses' location, their approach, and selected lane.

(vi) Detectors are needed throughout the area behind the stop line where buses can change their lane. These detectors also declare the existence of other cars and the bus's selected lane.

(vii) In the point VMS is installed, another detector is needed to count the private cars that occupied the distance between the VMS and the area behind the stop line.

(viii) The movement of the buses and cars is assumed static, and their speed value is constant, which is based on road rules.

3.2. The Model Procedure. The study aims to minimize the number of stops for optimizing the performance of the buses and private vehicles in urban arterials with signalized intersections equipped with presignals. Therefore, it is essential that

(i) buses be located in the first line of the queue behind the stop line; presignals are the appropriate methods for meeting this requirement by prioritizing buses behind intersections;

(ii) cars be at a moderate speed, without any stop or with the minimum stops behind presignals. To this end, it is required to minimize the initial queue behind presignals. More specifically, it would be necessary to minimize the initial queue delay [23] at presignals through the objective function proposed in (1).

Objective function:

$$
F(x)=\min \left[d_{3}\right]
$$

subject to

$$
\begin{aligned}
d_{3}= & \frac{1800 \times Q_{b} \times(1+u) \times t}{C T} \\
& \text { If } v_{\text {car,suggested }} \geq 5 \mathrm{~km} / \mathrm{h} \\
& \text { then } Q_{b}=0,
\end{aligned}
$$

where

$T$ is the duration of analysis period (h),

$Q_{b}$ is the initial queue at the start of period $T$ (veh),

$C$ is the adjusted lane group capacity (veh/h),

$t$ is the duration of unmet demand in $T(\mathrm{~h}),(t=0$ if $Q_{b}=0$, otherwise $t=\min \left[T, Q_{b} / C[1-\min (1, V / C)]\right]$ if $Q_{b} \neq 0$ ),

$u$ is the delay parameter $(u=0$ if $t<T$, otherwise $u=1-C T / Q_{b}[1-\min (1, V / C)]$ if $\left.t \geq T\right)$,

$v_{\text {car,suggested }}$ is the suggested speed of the car in arterials using the model $(\mathrm{km} / \mathrm{h})$.

It is required to identify some parameters in order to estimate the suggested speed of cars $\left(v_{\text {car,suggested }}\right)$. These parameters would be gained through the following three steps.

Step 1 (estimate the optimal distance for implementing presignals). At this distance, buses could select their desirable lane at the main intersection. Additionally, the AVL applied in buses declare their real-time position to private vehicles in order to reduce their speeds due to prioritizing the buses and their selected lane (shown in Figure 1 as $d_{\text {bus }}$ ).

There are several factors that need to be considered for the sake of estimating this distance. These factors are based on buses movements for changing their lane and reaching the stop line of the main intersection. In this paper, the distance of implementing presignals $\left(d_{\text {bus }}\right)$ is obtained by modifying the distance proposed by Guler and Menendez [21]. They assumed that bus stopped at the presignal so its initial speed to move towards the main intersection was zero. However, in this research, we suppose that buses do not stop at presignals and they reach the main intersection with an initial speed. Moreover, it is assumed that buses change their lane and arrive at the main intersection when traffic signal starts at red. Therefore, $d_{\text {bus }}$ can be calculated by the following equation:

$$
\begin{aligned}
d_{\text {bus }} & =\left[\left(c-r_{m s}\right)_{1} \times\left(\frac{C}{k_{\text {jam }}}-v_{1}\right)+\left(c-r_{m s}\right)_{2}\right. \\
& \left.\times\left(0-\frac{C}{k_{\text {jam }}}\right)\right] \times 1000,
\end{aligned}
$$

where

$d_{\text {bus }}$ is the distance between the presignal and the main intersection $(\mathrm{m})$,

$c$ is the common cycle length of the traffic signal (h),

$r_{m s}$ is the red duration at the traffic signal (h),

$C$ is the total capacity across all lanes at the main signal (veh/h), 


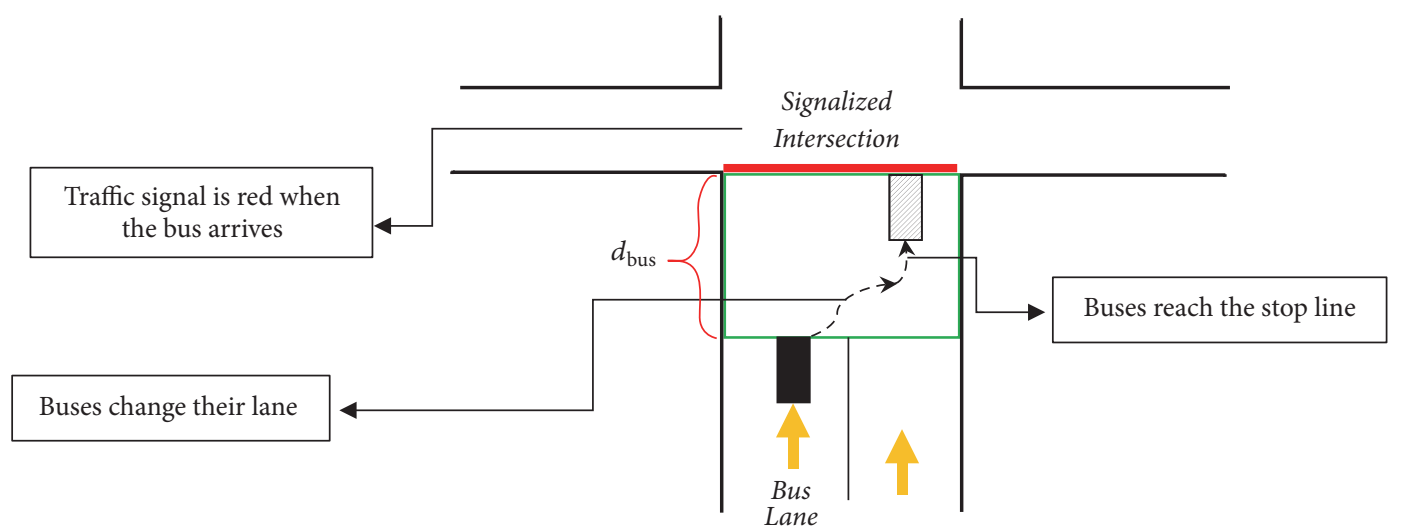

FIgURE 1: The buses' approach for changing lane and reaching the stop line.

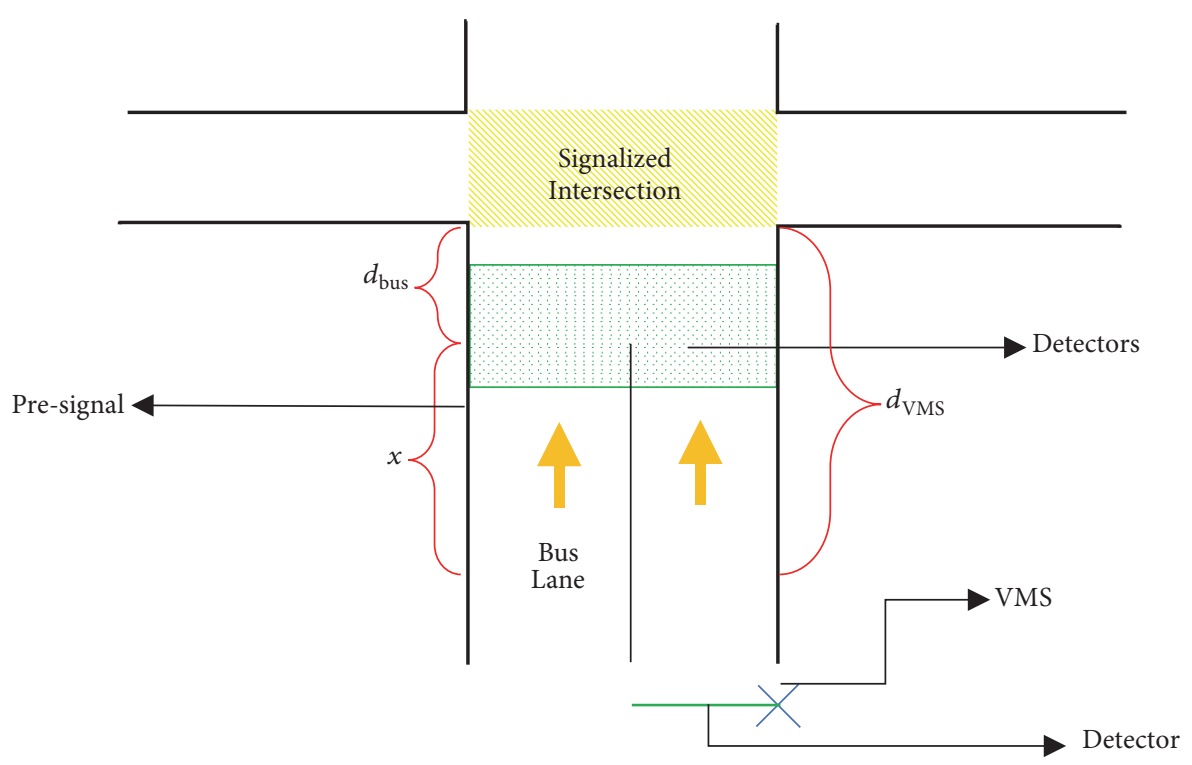

FIGURE 2: The schematic intersection components.

$k_{\text {jam }}$ is jam density (density at zero speed) (veh/ $\mathrm{km}$ ), $C / k_{\text {jam }}$ is the bus moving speed for changing lane and reaching the intersection,

$v_{1}$ is the bus initial speed, which is not zero,

$\left(c-r_{m s}\right)$ is the green duration of the traffic signal,

$\left(c-r_{m s}\right)_{1}$ is the required time for buses to change the lane with initial speed of $v_{1}$,

$\left(c-r_{m s}\right)_{2}$ is the required time for buses to reach the stop line with speed of $C / k_{\text {jam }}$.

Step 2 (determine the distance between cars and intersection for installing a VMS in order to give the car real-time information about its appropriate speed). The required distance lets the lead vehicle accelerate or decelerate its speed. In other words, it would be an essential distance for a car to moderate and justify its speed in the critical traffic situation. This distance (4) is related to the distance between bus and intersection, and the drivers' reaction time and their current speed. It should be considered that the distance of presignal $\left(d_{\text {bus }}\right)$ estimated in (3) has to be added to this distance. Its details are depicted in Figure 2.

To calculate the distance:

$$
\begin{aligned}
x & =\frac{v_{\text {current }} t_{\text {reaction }}}{3.6} \\
d_{\mathrm{VMS}} & =x+d_{\text {bus }},
\end{aligned}
$$

where

$v_{\text {current }}$ is the speed of the lead vehicle, which is assumed constant and based on the road rules $(\mathrm{km} / \mathrm{h})$, $t_{\text {reaction }}$ is the reaction time of the lead vehicle once deciding to change its speed (s), which is assumed $2 \mathrm{~s}$, $x$ is the distance between the lead vehicle and the presignal, 
$d_{\mathrm{VMS}}$ is the essential distance between the lead vehicle and the intersection which is required for the lead vehicle in the critical situation to stop the car (m).

Step 3 (optimize the speed of vehicles before reaching presignals for the sake of reducing their number of stops). In order to minimize the cars' number of stops, speed and initial queue delay are considered. Alleviating the private vehicles' stops is done through controlling the vehicles speed, which leads to eliminating the vehicles' initial queue delay. When the bus arrives at $d_{\text {bus }}$, cars would be declared about its lane changing. As mentioned above, cars get the alarm earlier in $d_{\mathrm{VMS}}$. Thus, cars get the chance to reduce their speed pertaining to several factors: buses travel time, speed, selected lane, $d_{\text {bus }}$, traffic signal timing, whether it is red or green, and the performance of the buses while crossing the intersection. Using the mentioned factors, the desired arrival time for cars is estimated. Then its speed can be calculated in accordance with the distance, $d_{\mathrm{VMS}}$.

In order to estimate the vehicle's speed and consequently the following vehicles' speed, the below equations are used:

$$
\begin{aligned}
t_{B} & =\max \left[t_{\text {bus }}, r_{m s}\right] \\
t_{\text {bus }} & =\frac{3.6 d_{\text {bus }}}{v_{\text {bus }}},
\end{aligned}
$$

where

$t_{B}$ is the bus's travel time according to traffic signal timing (s),

$t_{\text {bus }}$ is the bus's travel time (for changing its lane) to pass the area behind the stop line (s),

$d_{\text {bus }}$ is the distance between the presignal and the main intersection $(\mathrm{m})$,

$v_{\text {bus }}$ is the bus's mean speed of near the intersection $(\mathrm{km} / \mathrm{h})$,

$r_{m s}$ is the duration of red time in traffic signal (s).

$$
t_{\mathrm{car}}=\frac{3.6 d_{\mathrm{VMS}}}{v_{\text {car,current }}},
$$

$t_{\text {car }}$ is the car's travel time to reach the intersection (s), $d_{\mathrm{VMS}}$ is the proposed distance for installing the VMS (m),

$v_{\text {car,current }}$ is the car's mean speed in arterials $(\mathrm{km} / \mathrm{h})$.

$$
t_{C}=t_{B}+t_{\text {car }}
$$

$t_{C}$ is the car's travel time according to the leading traffic condition (s).

$$
v_{\text {car,suggested }}=\frac{d_{\mathrm{VMS}}}{t_{\mathrm{C}}} \times 3.6=v_{\text {lead }},
$$

$v_{\text {car,suggested }}$ is the suggested speed of the car in arterials according to the leading traffic condition, in terms of the existence of the buses changing their lanes and traffic signal timing $(\mathrm{km} / \mathrm{h})$.
The suggested speed for private vehicles estimated in (9), $v_{\text {car,suggested }}$ is the lead vehicle's speed. As a result, the private vehicles are informed of the traffic condition prior to reaching the intersection, and they are notified about the appropriate speed. Consequently, not only the number of stops for private vehicles would be reduced, but also the buses would be prioritized to select their approach.

The point is that the VMS should be installed in the optimum place to inform the private vehicles in an appropriate distance. This distance should be efficient in informing private vehicles even in the critical traffic condition. It should be considered that traffic condition is influenced by several factors which are as follows:

(i) Occupying the mentioned distance by private vehicles travel in front of the lead vehicle.

(ii) The bus, which travels in the bus lane, changes its lane in $d_{\text {bus }}$ distance, or keeps moving in its lane.

(iii) Considering time and type of traffic signal in terms of red or green and its duration.

The value of speed depicted in the VMS needs to be updated when the traffic signal changes from red to green or vice versa.

\section{Results and Discussion}

The proposed model would be analyzed numerically through an example of signalized intersection equipped with a presignal system for both before and after implementing the model. In addition, vehicles' delay could be estimated in the mentioned cases, and the desirability of the model would be demonstrated by comparing the results. For the sake of analyzing this model, some assumptions have been assumed which are presented in Table 1. More specifically, the signal cycle length is $60 \mathrm{~s}$, and the ratio of green time to cycle length $(g / c)$ is 0.5 . The density and capacity of the arterial are $100 \mathrm{veh} / \mathrm{km}$ and $800 \mathrm{veh} / \mathrm{h}$, respectively. In addition, the proportion of traffic flow to capacity $(V / C)$ is 1 . The speed of buses and cars is hypothetically $20 \mathrm{~km} / \mathrm{h}$ and $40 \mathrm{~km} / \mathrm{h}$ before implementing the model.

The essential distance for buses to change their lane before the intersection $\left(d_{\text {bus }}\right)$ and the required distance between the lead vehicle and the intersection for installing VMS $\left(d_{\mathrm{VMS}}\right)$ could be calculated using the mentioned parameters (assumed in Table 1) and the equations (proposed in the previous section). On the other hand, suggested speed and travel time of the cars would be estimated to be shown on VMS.

The most important difference between the current situation and applying the proposed model is forming a queue of vehicles behind presignals due to the red signal imposed on cars. This issue would cause initial queue delay in the case of implementing presignal without any modification. Consequently, there would be a remarkable increase in delay. In other words, implementing the model would eliminate the cars' stops behind the presignal. Therefore, the cars' delay and per person's delay related to additional stop would be alleviated. As it has been shown in Table 1, the delay has been reduced $20 \%$ through implementing the model in $V / C=1$ 
TABLE 1: Assumptions for parameters used in the model.

\begin{tabular}{|c|c|c|}
\hline Parameters & Values (current situation) & Values (after implementing the model) \\
\hline Duration & $1 \mathrm{~h}$ & $1 \mathrm{~h}$ \\
\hline Number of buses & 12 & 12 \\
\hline Number of cars $(V)$ & 800 veh & 800 veh \\
\hline Bus headway & $300 \mathrm{~s}$ & $300 \mathrm{~s}$ \\
\hline Car headway & $4.5 \mathrm{~s}$ & $4.5 \mathrm{~s}$ \\
\hline Cycle length $(c)$ & $60 s$ & $60 s$ \\
\hline Red duration $\left(r_{m s}\right)$ & $30 \mathrm{~s}$ & $30 s$ \\
\hline Capacity $(C)$ & $800 \mathrm{veh} / \mathrm{h}$ & $800 \mathrm{veh} / \mathrm{h}$ \\
\hline Jam density $\left(K_{\text {jam }}\right)$ & $100 \mathrm{veh} / \mathrm{km}$ & $100 \mathrm{veh} / \mathrm{km}$ \\
\hline Initial speed of bus $\left(V_{1 b}\right)$ & $20 \mathrm{~km} / \mathrm{h}$ & $20 \mathrm{~km} / \mathrm{h}$ \\
\hline Initial speed of car $\left(V_{1 c}\right)$ & $40 \mathrm{~km} / \mathrm{h}$ & $40 \mathrm{~km} / \mathrm{h}$ \\
\hline Green duration $(G)$ & $30 \mathrm{~s}$ & $30 \mathrm{~s}$ \\
\hline Time for bus to change lane & $15 \mathrm{~s}$ & $15 \mathrm{~s}$ \\
\hline Time for bus to reach the stop line & $15 s$ & $15 s$ \\
\hline Capacity/density & 8 & 8 \\
\hline$d_{\text {bus }}$ & $83.33 \mathrm{~m}$ & $83.33 \mathrm{~m}$ \\
\hline Distance of VMS for car $(x)$ & -- & $22.22 \mathrm{~m}$ \\
\hline Reaction time $(t)$ & $2 \mathrm{~s}$ & $2 \mathrm{~s}$ \\
\hline$d_{\mathrm{VMS}}$ & -- & $106 \mathrm{~m}$ \\
\hline Bus travel time $\left(t_{\text {bus }}\right)$ & $15 \mathrm{~s}$ & $15 \mathrm{~s}$ \\
\hline Suggested travel time of bus $\left(t_{B}\right)$ & $30 \mathrm{~s}$ & $30 \mathrm{~s}$ \\
\hline Car travel time $\left(t_{\text {car }}\right)$ & -- & $9.5 \mathrm{~s}$ \\
\hline Suggested travel time of car $\left(t_{C}\right)$ & -- & $39.5 \mathrm{~s}$ \\
\hline Suggested speed of car $\left(V_{\text {car, suggested }}\right)$ & -- & $9.62 \mathrm{~km} / \mathrm{h}$ \\
\hline Traffic flow $(V)$ & $800 \mathrm{veh} / \mathrm{h}$ & $800 \mathrm{veh} / \mathrm{h}$ \\
\hline$X(V / C)$ & 1.00 & 1.00 \\
\hline Initial queue $\left(Q_{(b)}\right)$ & $10 \mathrm{veh}$ & 0 veh \\
\hline Uniform delay $\left(d_{(1)}\right)$ & $15 \mathrm{~s}$ & $15 \mathrm{~s}$ \\
\hline Incremental delay $\left(d_{(2)}\right)$ & $64 s$ & $64 \mathrm{~s}$ \\
\hline Progression adjustment factor (PF) & 1.667 & 1.667 \\
\hline Initial queue delay $\left(d_{(3)}\right)$ & $22.5 \mathrm{~s}$ & $0 \mathrm{~s}$ \\
\hline Delay & $111.14 \mathrm{~s}$ & $88.64 \mathrm{~s}$ \\
\hline Travel time bus & $126.14 \mathrm{~s}$ & $103.64 \mathrm{~s}$ \\
\hline Travel time car & $150.64 \mathrm{~s}$ & $128.14 \mathrm{~s}$ \\
\hline$g / c$ & 0.5 & 0.5 \\
\hline Average car occupancy & $2 \mathrm{p}$ & $2 \mathrm{p}$ \\
\hline Delay/person & $55.57 \mathrm{~s} / \mathrm{pp}$ & $44.32 \mathrm{~s} / \mathrm{pp}$ \\
\hline
\end{tabular}

and $g / c=0.5$, and it would be an illustration for the efficient effect of the model on both cars' delay and per person's delay.

In order to conduct numerical analyses of the model, the fluctuation of $\mathrm{g} / \mathrm{c}$ and $\mathrm{V} / \mathrm{C}$ would be considered, and its influence on cars' delay would be determined. Figure 3 compares the amount of delay in the current situation and after implementing the model at $g / c=0.5$ and different $V / C$ ratios.

As is shown in Figure 3, implementing the proposed model has declined delay at all $V / C$ ratios. It has to be mentioned that the percentage of delay improvement is decreased as the ratio of $V / C$ increased. In other words, the reduction of delay is $63 \%$ at undersaturated condition, while it is $20 \%$ at oversaturated one.

\section{Conclusion}

One of the negatively influential issues regarding urban life is the traffic jam. It has negative impacts on people's life and well-being due to increasing their travel time and delay. It has to be mentioned that it would not only disturb the dwellers' planning and timetable, but also threaten individuals' health because of air pollution.

The suggested strategy to decrease traffic jam is to encourage people to use public transportation through improving its systems. One of these motivations is to give priority to this mode by implementing presignals behind the signalized intersections. Presignal is a signal that is installed in advance of the intersections and gives private cars red signal to 


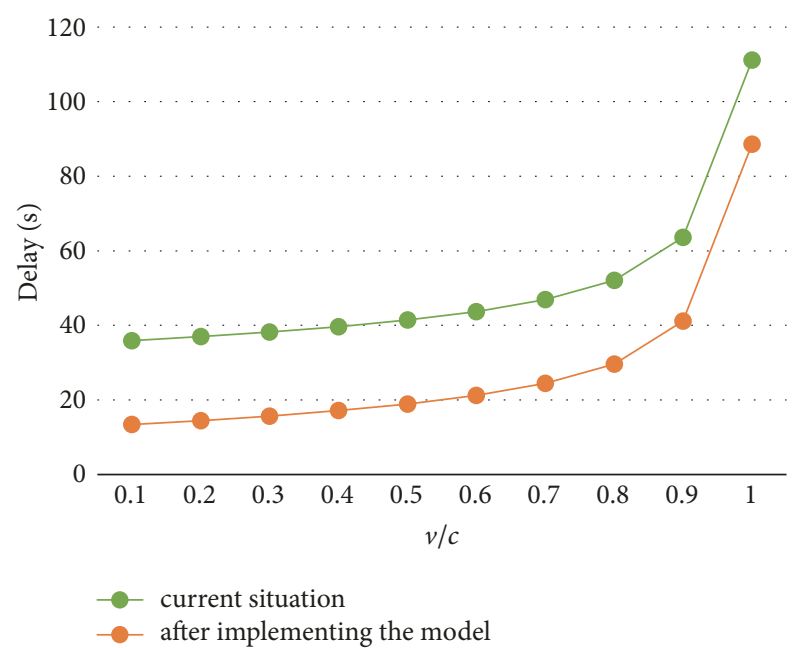

FIGURE 3: Comparison of private vehicles' delay for the current situation and the proposed presignal's model with different $V / C$ ratios at $g / c=0.5$.

prioritize buses in selecting their lane and approach behind the main intersection. By this way, conflicts between cars and buses while discharging the intersection are eliminated and the priority for locating in the first line of the queue and discharging the intersection is given to buses. It should be considered that as buses have much higher occupancies, the overall delay and travel time for passengers are decreased remarkably.

This strategy reduces buses' delay and travel time and increases their speed efficiently. However, cars are not taken into account in presignals, and they are given additional stops behind the presignal. More specifically, installing presignals enhance the functionality of buses by giving them priority and eliminating, or at least alleviating, the probable conflicts between them and cars. However, it worsens the performance of the private vehicles because of increasing their number of stops.

The study aims to develop this relatively modern method, presignals. In this research, it is considered to minimize the private vehicles' number of stops behind presignals. Thus, their travel time, delay, and speed are optimized. In other words, it is suggested to prioritize buses and develop cars' performance simultaneously by minimizing their number of stops behind presignals. For this purpose, it is proposed to balance the cars' speed according to buses' destination and traffic signal timing. Therefore, the delay of cars would be decreased remarkably.

\section{Conflicts of Interest}

The authors declare that they have no conflicts of interest.

\section{Acknowledgments}

The study is supported by the National Natural Science Foundation of China (no. 71701070) and the Natural Science Foundation of Guangdong Province (no. 2016A030310427).

\section{References}

[1] L. Han, D. Z. W. Wang, H. K. Lo, C. Zhu, and X. Cai, "Discretetime day-to-day dynamic congestion pricing scheme considering multiple equilibria," Transportation Research Part B: Methodological, vol. 104, pp. 1-16, 2017.

[2] Y. Kuang, X. Qu, and S. Wang, "Propagation and dissipation of crash risk on saturated freeways," Transportmetrica B, vol. 2, no. 3, pp. 203-214, 2014.

[3] D. Z. W. Wang and B. Du, "Continuum modelling of spatial and dynamic equilibrium in a travel corridor with heterogeneous commuters-A partial differential complementarity system approach," Transportation Research Part B: Methodological, vol. 85, pp. 1-18, 2016.

[4] Z. Liu, S. Wang, B. Zhou, and Q. Cheng, "Robust optimization of distance-based tolls in a network considering stochastic day to day dynamics," Transportation Research Part C: Emerging Technologies, vol. 79, pp. 58-72, 2017.

[5] X. Qu, S. Wang, and J. Zhang, "On the fundamental diagram for freeway traffic: a novel calibration approach for single-regime models," Transportation Research Part B: Methodological, vol. 73, pp. 91-102, 2015.

[6] Z. Liu, Y. Yan, X. Qu, and Y. Zhang, "Bus stop-skipping scheme with random travel time," Transportation Research Part C: Emerging Technologies, vol. 35, pp. 46-56, 2013.

[7] A. Nayan and D. Z. W. Wang, "Optimal bus transit route packaging in a privatized contracting regime," Transportation Research Part A: Policy and Practice, vol. 97, pp. 146-157, 2017.

[8] Y. Bie, D. Wang, and X. Qu, "Modelling correlation degree between two adjacent signalised intersections for dynamic subarea partition," IET Intelligent Transport Systems, vol. 7, no. 1, pp. 28-35, 2013.

[9] Z. Liu, S. Wang, W. Chen, and Y. Zheng, "Willingness to board: a novel concept for modeling queuing up passengers," Transportation Research Part B: Methodological, vol. 90, pp. 7082, 2016.

[10] Q. Meng and X. Qu, "Bus dwell time estimation at bus bays: a probabilistic approach," Transportation Research Part C: Emerging Technologies, vol. 36, pp. 61-71, 2013.

[11] Y. Bie, Z. Liu, and Y. Wang, "A real-time traffic control method for the intersection with pre-signals under the phase swap sorting strategy," PLoS ONE, vol. 12, no. 5, Article ID 0177637, 2017.

[12] M. Peake, "Getting buses to the front of the queue," in Proceedings of the IPENZ Transportation Conference, Queenstown, New Zealand, 2006.

[13] J. Wu and N. Hounsell, "Bus priority using pre-signals," Transportation Research Part A: Policy and Practice, vol. 32A, no. 8, pp. 563-583, 1998.

[14] S. S. P. Kumara and N. B. Hounsell, "Bus priority and queue relocation capabilities of pre-signals: an analytical evaluation," in Proceedings of the 11th IFAC Symposium on Control in Transportation Systems, pp. 391-396, Delft, The Netherlands, 2006.

[15] H. He, S. I. Guler, and M. Menendez, "Adaptive control algorithm to provide bus priority with a pre-signal," Transportation Research Part C: Emerging Technologies, vol. 64, pp. 28-44, 2016.

[16] Y. Xuan, Increasing the flow capacity of signalized intersections with pre-signals: theory and case study [PhD thesis], University of California Berkeley, 2011.

[17] Y. Xuan, M. J. Cassidy, and C. F. Daganzo, "Using a pre-signal to increase bus- and car- carrying capacity at intersections: theory and experiment," in TRB 2012 Annual Meeting, 2011. 
[18] L. Kejun, "Bus priority signal control at isolated intersection," in International Conference on Intelligent Computation Technology and Automation, vol. 2, pp. 234-237, IEEE Computer Society, Montpellier, France, 2008.

[19] H. He, S. I. Guler, and M. Menendez, "Providing bus priority using adaptive re-signals," Online Pre-Signal Strategies TRB Final after Reviews, 2014, http://docs.trb.org/prp/15-2793.pdf.

[20] S. I. Guler and M. Menendez, "Analytical formulation and empirical evaluation of pre-signals for bus priority," Transportation Research Part B: Methodological, vol. 64, pp. 41-53, 2014.

[21] S. I. Guler and M. Menendez, "Pre-signals for bus priority: basic guidelines for implementation," Public Transport, vol. 7, no. 3, pp. 339-354, 2015.

[22] S. I. Guler, V. V. Gayash, and M. Menendez, "Bus priority at signalized intersections with single-lane approaches: A novel pre-signal strategy," Transportation Research Part C: Emerging Technologies, vol. 63, pp. 51-70, 2016.

[23] Highway Capacity Manual: Metric Units, Transportation Research Board, Washington, DC, USA, 2000. 


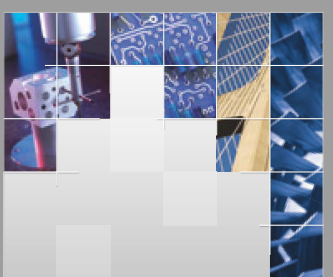

\section{Enfincering}
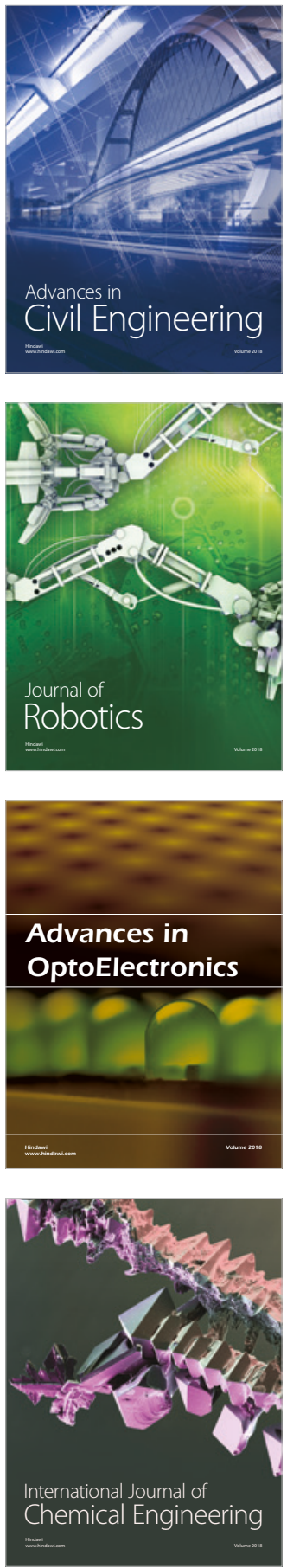

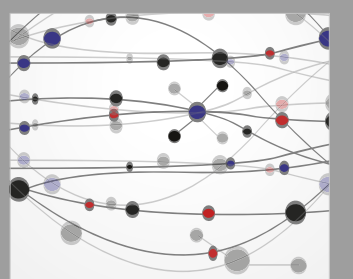

\section{Rotating \\ Machinery}

The Scientific World Journal

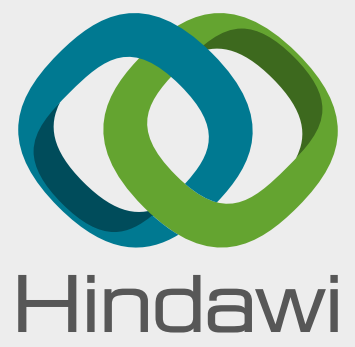

Submit your manuscripts at

www.hindawi.com
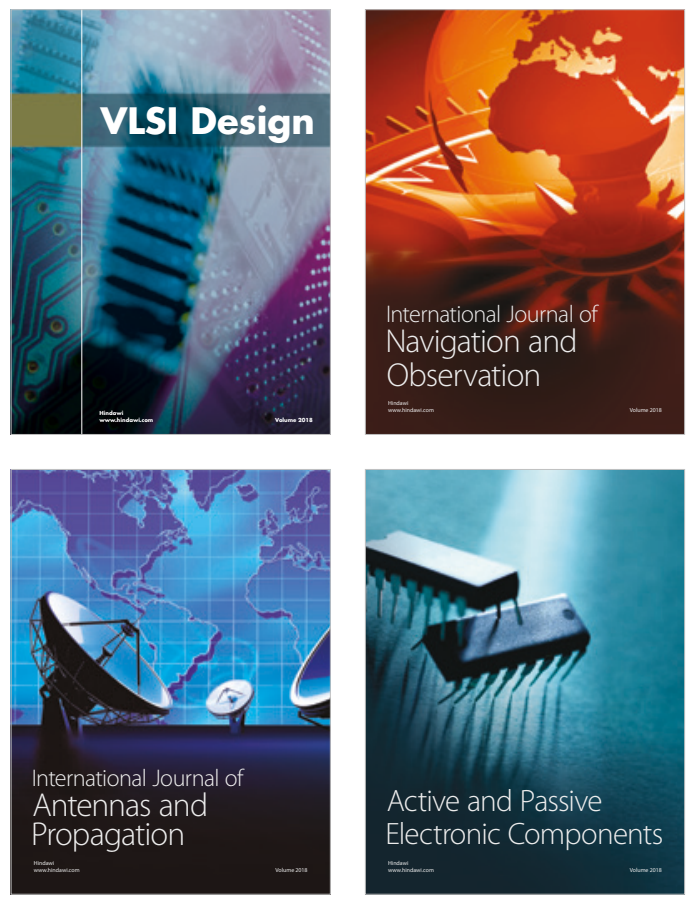
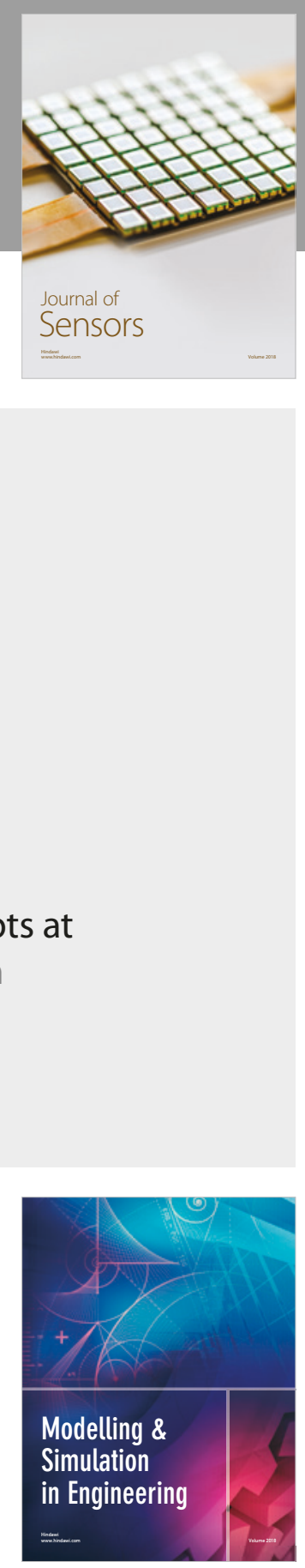

\section{Advances \\ Multimedia}
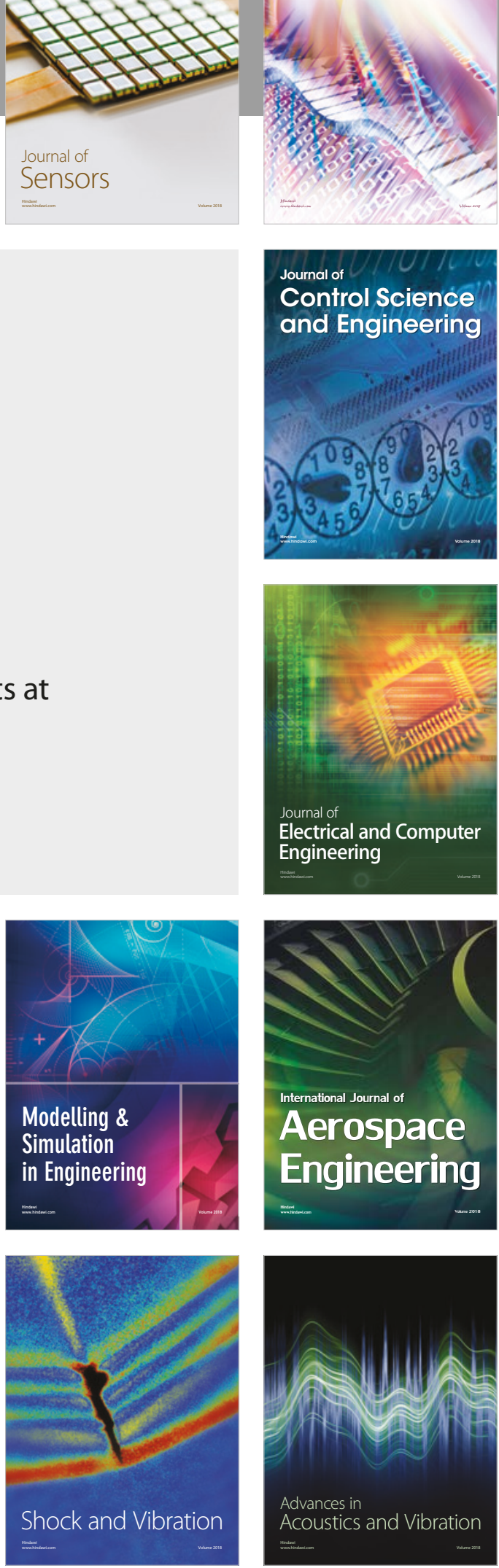